\title{
Combination of ISOM and K-SVD based on Dictionary Incremental Learning for Image Signal Processing
}

\author{
Guixin Sun ${ }^{1, a}$ \\ The People's Liberation Army Unit 91550, Dalian, 116023, China \\ aemail: GuixinSDLI@163.com
}

Keywords: Incremental Self-Organizing Maps; K-SVD; Dictionary Incremental Learning

\begin{abstract}
In this paper, a dictionary incremental learning method combination of ISOM(Incremental Self-Organizing Maps) and K-SVD is proposed. This method significantly improves the efficiency of K-SVD method for large sample based on the incremental learning ability of ISOM network. The experiment of image super resolution reconstruction shows that the proposed method has the capability of incremental learning.
\end{abstract}

\section{Introduction}

Signal sparse representation is one of hot topics in image signal processing, which has been applied successfully in several areas of image signal processing [1] [2]. Dictionary learning is an important part of the sparse representation. The K-SVD [3] dictionary learning algorithms for its simple and efficient features become research priorities. All K-SVD dictionary learning algorithms are two way optimization of iterative batch processing, in additional words, in every iteration, all training set will be accessed, and under constraints, minimize cost function, amount of calculation would be huge, not convenient for or barely to handle big sample sets.

Therefore, this paper introduces a dictionary incremental learning method combination of ISOM (Incremental Self-Organizing Maps) and K-SVD. This method will firstly divide a big sample set into a main training set and several sub training sets, then learn initial dictionary from major training set by K-SVD, later learning incremental dictionary from sub training sets. It will combine dictionaries based on ISOM, interactively process all sub training sets, to complete learning on big samples sets. This algorithm, compared with whole process, will cost lower internal memory, lower calculation workload as benefits, and meaningful for sparse representation of big sets on program application.

\section{ISOM Introduction}

The traditional SOM has a fixed structure, in other words, the network is unable to acquire new knowledge without destroying previously acquired knowledge structures. For this reason, some incremental learning algorithms with single self-organizing layer have been proposed [4][5][6], these methods remove the need for prior specification of the network architecture but assume that the pattern set is finite. Robert [7] proposed a plastic self-organizing map, but it can't preserve topographical neighbors. Yann [8] proposed an incremental and growing network model which is able to learn the topological relations in a given set of input vectors. However, its labeling system algorithm needs the user to correct the network's learning. Khalid [9] presented a new method which built a first topology by SOM algorithm using the available sample set andupdating it when the new samples are inputted. In this paper, we combine paper's method [9] and traditional K-SVD, make traditional K-SVD algorithm with incremental learning ability to handle big sample sets learning.

\section{Description of Combination of ISOM and K-SVD}

Detailed algorithm is demonstrated as below. 


\section{Step 1:}

Initialize parameter, $t=2, \operatorname{dis}(x, y)$ is the Euclid distance between vector $x$ and $y$. The entire samples are divided into a main training set $Y_{1}$ and several sub training sets $Y_{2} \ldots Y_{\mathrm{N}}$. The sample number of main training set is more than sub training set.

Step2:

Obtain the dictionary $D_{0}$ and parameter matrix $V_{0}$ from the main training set $Y_{1}$ by K-SVD algorithm.

Step 3:

Obtain the dictionary $D_{\mathrm{t}}$ and parameter matrix $V_{\mathrm{t}}$ from the sub training set $Y_{\mathrm{t}}$ by K-SVD algorithm.

Step4:

Present the samples of pattern $X_{i}=D_{0}$ to the input layer of ISOM, and normalize it.

$$
\overline{X_{i}}=\frac{\left(x_{1}^{i}, x_{2}^{i}, \ldots, x_{n}^{i}\right)}{\sqrt{\left[x_{1}^{i}\right]^{2}+\left[x_{2}^{i}\right]^{2}+\ldots+\left[x_{n}^{i}\right]^{2}}}
$$

Step5:

Select connecting weight vector, $W_{j}=V_{0}$, and normalize it. Compute the Euclid distance between $\overline{X_{i}}$ and $\overline{W_{j}}$.

$$
\begin{aligned}
& \overline{w_{j}}=\frac{w_{j}}{\left\|w_{j}\right\|}=\frac{\left(w_{j 1}, w_{j 2}, \ldots, w_{j n}\right)}{\sqrt{\left|w_{j 1}\right|^{2}+\left|w_{j 2}\right|^{2}+\ldots+\left|w_{j n}\right|^{2}}} \\
& d_{j}=\sqrt{\sum_{k=1}^{n}\left(x_{k}^{i}-\overline{w_{j k}}\right)^{2}}, j=1,2, \ldots, M
\end{aligned}
$$

Step6:

Find the minimum distance $d_{\mathrm{g}}$, determine the winning neuron $g$. Modify the connecting weight vectors between the neurons of input layer and the ones in the neighborhood $N_{\mathrm{g}}(c)$ of competitive layer. Where $\eta(c)$ denotes the learning rate at the time $c$.

$$
\left.\overline{w_{i j}(c+1)}=\overline{w_{i j}(c)}+\eta(c) \boldsymbol{g} x_{i}^{k}-\overline{w_{i j}(c)}\right]
$$

Step7:

Update the topology of ISOM, present the sub training set dictionary $D_{\mathrm{t}}$ and parameter matrix $V_{\mathrm{t}}$ to the initial map of ISOM. That classifies the new elements in the map. Then, we only select the winning neurons which are situated in the area of the grid and have a between the elements falling into the $N_{\mathrm{g}}(t)$ and all elements of the new sample set. Initialize the weight of the new neuron $j^{\prime}$.

$$
W_{j}^{\prime}(k)=\frac{1}{2}\left(\frac{1}{N^{\prime}} \sum_{i=1}^{N^{\prime}} X_{i}(k)+W_{j}(k)\right)
$$

$W_{j}^{\prime}(k)$ is the $k$ th element of weight vector of the new neuron which is adjacent to the selected neuron $j^{\prime} . N^{\prime}$ is the size of the sub training set. $X_{i}(k)=D_{\mathrm{t}}(k)$ is the weight vector of the sub training set. $W_{\mathrm{j}}(k)=V_{\mathrm{t}}(k)$ is the weight vector of neuron $j$ after learning of the initial map.

Step8:

Modify $C$, which is similar to Step (6).Optimize the structure of the network.

Step 9:

Let $t=t+1$, return Step (3), until $t=p$. Record each weight vector $W_{\mathrm{i}} *$ of every winning neuron, let $W_{\mathrm{g}}$ represents the set of weight vectors of the winning neurons. $W_{\mathrm{g}}=\left\{\mathrm{W}_{\mathrm{i}}^{*}\right\}$ is the result of dictionary learning.

\section{Experiment and Results}

Select the images with different structure and texture, such as Figure 1. By pre-degradation and extraction of high frequency component, append 1 variance $3 \times 3$ Gaussian Blur , 2 variance additive noise , 3-desample Bicubic, establish training sample set with $680003 \times 3$ image blocks finally. Divide the sample set into 1 main training set and 4 sub training sets. The dictionary result of K-SVD, and incremental learning algorithm proposed in this paper. The result of super-resolution 
reconstruction with the algorithm[10], using above different dictionaries, is shown in Figure 2 . The result of time-consuming comparing above two algorithms is shown in Table 1.
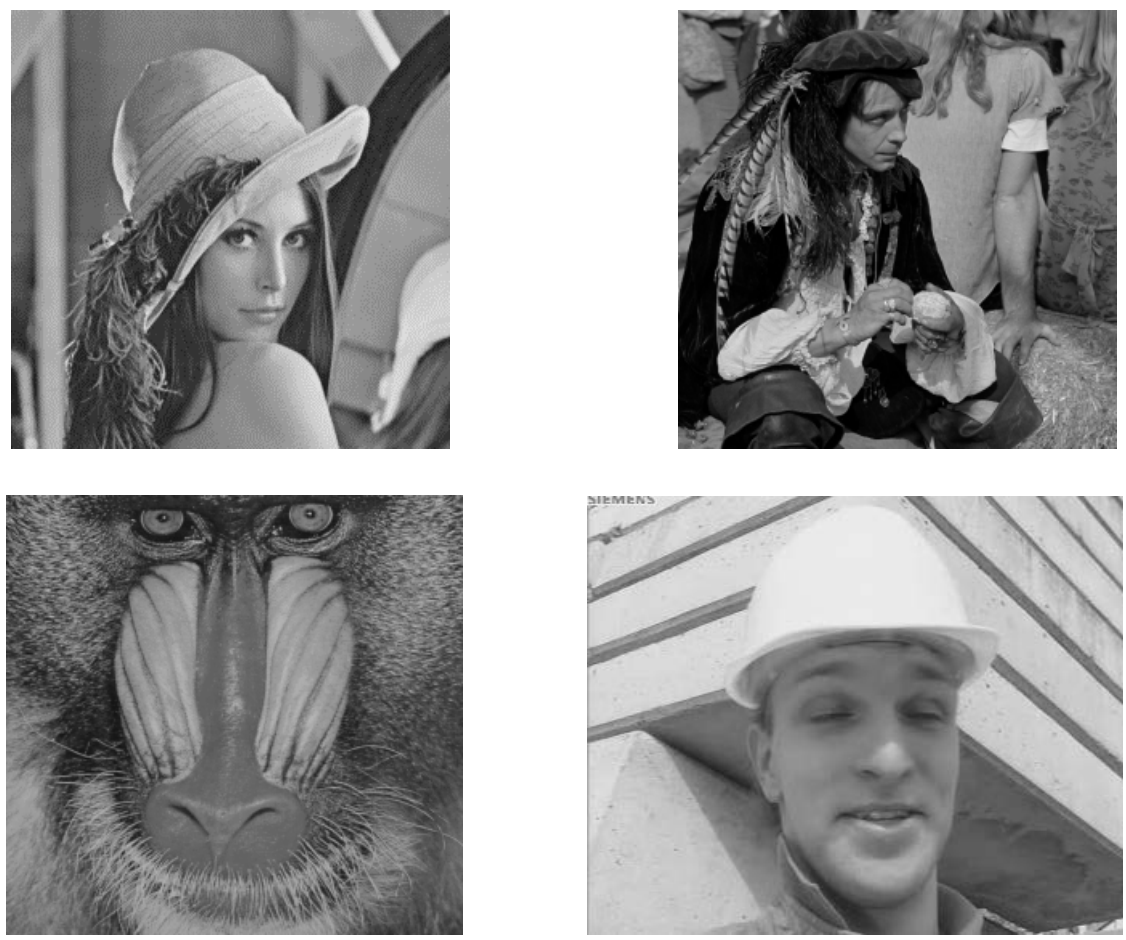

Fig.1. Training samples of experiment images with lenna, man, baboon, foreman
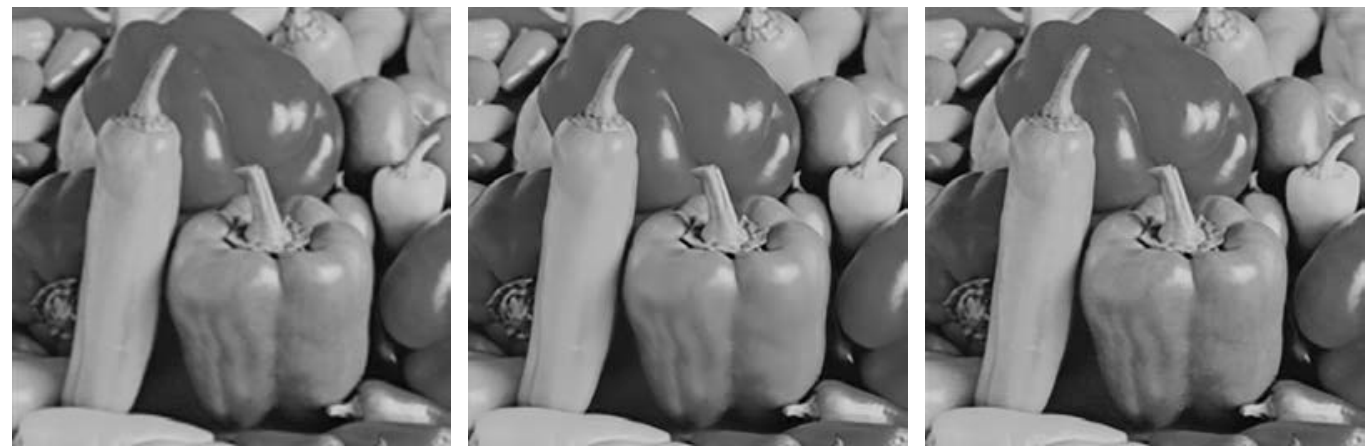

Fig.2. Result of SRR with Bicubic(left), K-SVD(middle), the method proposed in this paper(right)

Tab.1. Result of time-consuming.

\begin{tabular}{ccc}
\hline & K-SVD & Incremental learning K-SVD \\
\hline Establish Dictionary duration(s) & 2185 & 547 \\
& & \\
\hline
\end{tabular}

\section{Conclusion}

As shown in Table 1, compared with traditional K-SVD, the dictionary incremental learning method combination of ISOM and K-SVD can obtain the capability of incremental learning and better performance with time-consuming. Therefore the algorithm proposed in this paper can cost lower internal memory, lower calculation workload as benefits, and meaningful for sparse representation of big sets on program application.

\section{References}

[1] M. Elad, M. Aharon. Image denoising via sparse and redundant representations overlearned dictionaries. Image Processing, IEEE Transactions on, 2006,15(12):3736-3745. 
[2] M. Elad, J.L. Starck, P. Querre. Simultaneous cartoon and texture image inpainting using morphological component analysis (MCA). Applied and ComputationalHarmonic Analysis, 2005,19(3): 340-358.

[3] M. Aharon, M. Elad, A. M. Bruckstein. The K-SVD: An algorithm for designing ofovercomplete dictionaries for sparse representation. IEEE Transactions on SignalProcessing, 2006,54(11): 4311-4322.

[4] Friake B. Growing cell structures-self organizing network for unsupervised and supervised learning[J]. Neural Networks, 1994,7(9):1441-1460.

[5] Friake B. Growing grid-a self-organizing network with constant neighbourhood range and adaptation strength[J]. Neural Processing Letter, 1995, 2(5):9-13.

[6] Friake B. A growing neural gas learns topologies[C]. Advances in neural information processing systems 7:Proceedings of the 1994 Conference on Neural Information Processing Systems.Cambridge, MA:MIT Press, 1995,632-635.

[7] Lang R, Warwick K. The plastic self organising map[C]. Proceedings of the 2002 International Joint Conference on Neural Networks. Honolulu, HI, USA, 2002, 727-732.

[8] Prudent Y, Ennaji A. An incremental growing neural gas learns topologies[C]. Proceedings of International Joint Conference on Neural Networks. Montreal, Canada, 2005, 1211-1216.

[9] Benabdeslem K, Bennani Y. An incremental SOM for web navigation patterns clustering[C]. 26th International Conference on Information Technology Interfaces. SRCE-University Computing Centre, University of Zagreb, Croatia. Cavtat / Dubrovnik, Croatia, 2004, 209-213.

[10] S. Park, M. Park, M. G. Kang. Super-resolution image reconstruction: a technical overview. IEEE Signal Processing Magazine, 2003,20(3): 21-36. 